\title{
Drug Use Evaluation of Sitagliptin Dosing by Pharmacist Versus Nonpharmacist Clinicians in an Internal Medicine Department of a Private Physician-Owned Multispecialty Clinic
}

\author{
M. Shawn McFarland, PharmD; L. Brian Cross, PharmD; Benjamin Gross, PharmD; \\ Chad Gentry, PharmD; Jeffrey Tunney, PharmD; and U.P. Patel, MD
}

\begin{abstract}
BACKGROUND: Sitagliptin is recommended for initial and maintenance dosing at $100 \mathrm{mg}$ daily. Downward dose adjustment is recommended in patients with moderate or severe renal insufficiency.

OBJECTIVE: To determine the prevalence of the potentially inappropriate initial dosing of sitagliptin based on estimated glomerular filtration rate (GFR) at baseline for pharmacist versus nonpharmacist prescribers in an internal medicine department of a private physician-owned multispecialty clinic that included a pharmacist-managed diabetes program.

METHODS: This was a retrospective cross-sectional cohort analysis using data from an electronic medical record database of a private physicianowned multispecialty clinic that included a pharmacist-managed diabetes program. For patients prescribed sitagliptin between 0ctober 17, 2006, and June 5,2008 , the variables of interest were (a) the initial sitagliptin dose; (b) the GFR, calculated for each patient using the 4-point Modification of Dosing in Renal Disease (MDRD) formula at the time of initiation of sitagliptin; and (c) whether the clinician initiating the dose was a pharmacist or nonpharmacist (i.e., internal medicine physician, nurse practitioner, or physician assistant).

RESULTS: Of the 290 patients prescribed sitagliptin for the first time between 0ctober 17, 2006, and June $5,2008,35(12.1 \%)$ received a potentially inappropriate initial dose according to product labeling regarding renal function; 21 were over-dosed and 14 were under-dosed. Potentially inappropriate dosing occurred in 1 of 158 patients $(0.6 \%)$ who had initial dosing prescribed by a pharmacist compared with 34 of 132 patients (25.8\%) for nonpharmacists $(P<0.001$, Fisher's exact test).

CONCLUSION: Potentially inappropriate initial dosing of sitagliptin based on assessment of renal function was more likely to occur with nonpharmacist prescribers than with a pharmacist prescriber.
\end{abstract}

J Manag Care Pharm. 2009;15(7):563-67

Copyright $\odot$ 2009, Academy of Managed Care Pharmacy. All rights reserved.

\section{What is already known about this subject}

- There have been no studies reported in the medical literature regarding appropriate prescribing of sitagliptin based on label prescribing recommendations for renal function.

- The actual patient harm or effect on clinical biomarkers such as Alc associated with failure to adjust the dose of sitaglipitin based on renal function are unknown.

\section{What this study adds}

- Based on assessment of renal function, pharmacist prescribing of the initial dose of sitagliptin was associated with 1 case $(0.6 \%)$ of potentially inappropriate prescribing (under-dose) compared with 34 cases of potentially inappropriate prescribing (21 overdose and 13 under-dose) by nonpharmacists. The magnitude of the threat to patient safety or other clinical outcomes from this potentially inappropriate initial prescribing of sitagliptin is unknown.

$\mathrm{T}$ ype 2 diabetes affects more than 23.6 million people in the United States alone. In 2007, total excess cost for diabetes totaled $\$ 174$ billion. ${ }^{1}$ Since the U.S. approval of metformin, multiple treatments have been discovered and implemented that have had a direct impact on the control of type 2 diabetes. Appropriate selection and use of these agents is paramount to successful treatment of the type 2 diabetic patient.

On October 17, 2006, the U.S. Food and Drug Administration approved sitagliptin (Januvia), the first oral dipeptidyl peptidase IV inhibitor (DPP-IV) for the treatment of type 2 diabetes. ${ }^{2}$ DPP-IV inhibitors are enhancers of incretin hormones, particularly glucagon-like peptide 1 (GLP-1), which stimulates postprandial insulin secretion, increases satiety, decreases gastric emptying, and inhibits glucagon secretion. GLP-1 is excreted into the circulation by the enteroendocrine $\mathrm{L}$ cells after a meal but is rapidly inactivated by DPP-IV. Long-term treatment with sitagliptin, which inhibits the degradation of GLP-1 by DPP-IV, has been shown to reduce glycosylated hemoglobin levels, fasting glucose levels, and postprandial glucose excursions..$^{3-6}$ Sitagliptin is indicated as an adjunct to diet and exercise to improve glycemic control in adults with type 2 diabetes. Sitagliptin should not be used in patients with type 1 diabetes or for the treatment of diabetic ketoacidosis, and sitagliptin has not been studied in combination with insulin. ${ }^{7}$ Sitagliptin is available in 25 milligram (mg), $50 \mathrm{mg}$, and $100 \mathrm{mg}$ tablets

Multiple studies have been performed evaluating the pharmacokinetic properties of sitagliptin. ${ }^{8-11}$ Renal excretion is the primary mechanism of elimination for sitagliptin. Increases in sitagliptin area under the curve (AUC) have been shown to be less than 2-fold for patients with creatinine clearance $(\mathrm{CrCl})$ 


\section{Drug Use Evaluation of Sitagliptin Dosing by Pharmacist Versus Nonpharmacist Clinicians in an Internal Medicine Department of a Private Physician-Owned Multispecialty Clinic}

between 50-80 milliliters (ml) per minute; 2.3-fold higher for $\mathrm{CrCl} \geq 30-50 \mathrm{ml}$ per minute; 3.8-fold higher for $\mathrm{CrCl}<30 \mathrm{ml}$ per minute; and 4.5-fold higher for patients with end stage renal disease (ESRD). ${ }^{12}$ For this reason, sitagliptin dose adjustments are recommended by the manufacturer in the presence of renal dysfunction. Patients having $\mathrm{CrCl}$ of $>50 \mathrm{ml}$ per minute should receive a dose of $100 \mathrm{mg}$ per day; patients with $\mathrm{CrCl} \geq 30-50 \mathrm{ml}$ per minute should receive $50 \mathrm{mg}$; and patients with $\mathrm{CrCl}<30 \mathrm{ml}$ per minute should receive $25 \mathrm{mg}$ of sitagliptin. ${ }^{7}$ Recent data suggest that sitagliptin is well tolerated and provides effective glycemic control in patients with type 2 diabetes with moderate to severe renal insufficiency, including patients on dialysis. ${ }^{13}$ To our knowledge, no data exist examining the potentially inappropriate dosing of sitagliptin based on manufacturer guidelines.

The primary objective of this study was to evaluate the prevalence of the potentially inappropriate initial dosing of sitagliptin using manufacturer recommendations at the time of first initiation using the 4-point Modification in Diet and Renal Disease (MDRD) formula as an estimate of creatinine clearance in an internal medicine department. The 4-point MDRD formula estimates glomerular filtration rate (GFR) using the serum creatinine, age, gender, and ethnicity of the patient:

GFR $(\mathrm{mL} / \mathrm{min} / 1.73 \mathrm{~m} 2)=175 \times(\mathrm{Scr})-1.154 \times($ Age $)-0.203 \mathrm{x}$

(0.742 if female) $\mathrm{x}\left(1.212\right.$ if African American) ${ }^{14}$

The secondary objective was to evaluate the prevalence of potentially inappropriate dosing in patients for whom sitagliptin was initiated by a pharmacist versus patients in whom sitagliptin was initiated by a nonpharmacist prescriber.

This study received exempt status from the institutional review board (IRB) of the University of Tennessee Health Science Center.

\section{Methods}

This study was conducted in the internal medicine department (consisting of 10 physicians, 4 nurse practitioners, 1 physician assistant, and 1 clinical pharmacist) within a private physicianowned multispecialty clinic of 62 physicians. The internal medicine department averaged 423 patient visits per week. The clinic patients were approximately 38\% Medicare, 7\% Medicaid, 54\% commercial insurance, and 1\% cash-pay. The internal medicine department developed a pharmacist-directed diabetes disease management program in 2000. The Diabetes Care Clinic was established as a way to standardize the treatment of the type 2 diabetic patient within the internal medicine department by incorporating evidence-based medicine with a goal of achieving the standards of care established by the American Diabetes Association. The pharmacist-directed diabetes disease management program was voluntary, with patient enrollment initiated when poor diabetic control was documented by a nonpharmacist provider (i.e., physician, physician assistant, or nurse practitioner).

The pharmacist, who worked under a collaborative practice agreement, was responsible for evaluation and treatment of diabetes and conditions associated with diabetes. The pharmacist had the authority to order laboratory tests, initiate medications, make referrals, and schedule follow-up when deemed necessary. The pharmacist in this clinic saw an average of 20 diabetic patients per day for 4 and a half days per week. Patients not seen in the diabetes disease management program received usual medical care for their type 2 diabetes from their nonpharmacist provider.

This was a retrospective cross-sectional cohort analysis of all patients who received initial sitagliptin dosing by a provider in the internal medicine department from October 17, 2006, through June 5, 2008. Initial dosing of sitagliptin was defined as the first time an order for sitagliptin was entered in the electronic medical record. Since changing to electronic medical records in 2003, handwritten prescriptions were not allowed. Every prescription for any medication had to be entered into the electronic medical record before a prescription could be validated and given to the patient. Data were extracted from the electronic medical records database. Data evaluated for GFR were recorded for the present study if determined at the time of initiation of sitagliptin, or if not available, a GFR within 60 days prior to initiation of sitaglipitin was allowed. The MDRD equation was used to estimate renal function because the MDRD formula is now recommended by the National Kidney Foundation to estimate GFR for staging of chronic kidney disease (CKD). ${ }^{14}$ The MDRD equation was utilized for this study versus other methods to estimate renal function, because the source laboratory would report a GFR using the MDRD calculation on all reported basic metabolic profiles in an attempt to proactively evaluate and stage CKD. ${ }^{15}$ Estimation of renal function using other methods, such as the Cockroft-Gault equation, did not frequently occur at our facility.

Only patients for whom sitagliptin was initiated by a provider in the department of internal medicine were included in this study.

\section{Results}

Of 290 patients who received initial dosing of sitagliptin between October 17, 2006, and June 5, 2008, 13 patients (4.5\%) received $25 \mathrm{mg}$; 29 patients (10.0\%) received $50 \mathrm{mg}$; and 248 patients (85.5\%) received $100 \mathrm{mg}$ (Table 1). Appropriate dose according to label instructions regarding renal function was found for 255 patients (87.9\%, Table 2). For the 35 patients (12.1\%) with potentially inappropriate initial dosing of sitagliptin, 21 were over-dosed and 14 were under-dosed (Table 2).

There were 158 patients for whom sitagliptin was prescribed by a pharmacist, of which only 1 patient in the group with GFR $>50 \mathrm{ml}$ per minute was not prescribed the appropriate dose (100mg, Table 2). There were 132 patients for whom sitagliptin was prescribed by a nonpharmacist, of which 34 patients (25.8\%) were prescribed sitagliptin doses that did not correspond to the label recommendations based on GFR values. 
Drug Use Evaluation of Sitagliptin Dosing by Pharmacist Versus Nonpharmacist Clinicians

in an Internal Medicine Department of a Private Physician-Owned Multispecialty Clinic

\section{TABLE 1 Initial Doses of Sitagliptin According} to Glomerular Filtration Rate

\begin{tabular}{l|c|c|c|c}
\hline $\mathbf{n}=\mathbf{2 9 0}$ & $\mathbf{2 5 \mathbf { m g }}$ & $\mathbf{5 0 \mathbf { m g }}$ & $\mathbf{1 0 0 \mathbf { m g }}$ & Total \\
\hline GFR $<30$ & $8(66.7 \%)^{\mathrm{a}}$ & $1(8.3 \%)$ & $3(25.0 \%)$ & $12(100 \%)$ \\
\hline $\begin{array}{l}\text { GFR between } \\
30 \text { and } 50\end{array}$ & $1(5.7 \%)$ & $18(51.4 \%)^{\mathrm{a}}$ & $16(45.7 \%)$ & $35(100 \%)$ \\
\hline GFR $>50$ & $4(1.6 \%)$ & $10(4.1 \%)$ & $229(94.2 \%)^{\mathrm{a}}$ & $243(100 \%)$ \\
\hline Total & $13(4.5 \%)$ & $29(10.0 \%)$ & $248(85.5 \%)$ & 290 \\
\hline
\end{tabular}

aThe grey-shaded boxes identify appropriate dosing of sitaglipitin adjusted for renal function according to the product label instructions: 100 milligrams ( $\mathrm{mg}$ ) per day for patients with creatine clearance $(\mathrm{CrCl})>50$ milliliters $(\mathrm{ml})$ per minute, $50 \mathrm{mg}$ per day for patients with $\mathrm{CrCl} \geq 30-50 \mathrm{ml}$ per minute, and $25 \mathrm{mg}$ per day for patients with $\mathrm{CrCl}<30 \mathrm{ml}$ per minute. Glomerular filtration rate (GFR) was determined using the 4-point Modification of Dosing in Renal Disease equation. ${ }^{7}$

\section{Discussion}

Treatment of diabetes has become multifaceted and increasingly more complicated. The choice of treatment should be based on current available guidelines with the initial treatment composed of diet and metformin as well-validated core (tier 1) therapy. ${ }^{16}$ Additional treatment (tier 2) is left up to provider choice with options including sulfonylurea, pioglitazone, basal insulin, or GLP-1 enhancers. DPP-4 inhibitors such as sitagliptin are currently considered to be "other therapy."16 The use of sitagliptin may be an attractive option for some patients given its glucosedependent mechanism of action and weight neutrality compared with some other agents. ${ }^{6}$

Limited safety data currently exist regarding the use of sitagliptin. The most common side effects associated with sitagliptin are nasopharyngitis, urinary tract infections, headache, and gastrointestinal disturbances, with the latter seen more commonly with higher doses..$^{3-5}$ Postmarket hypersensitivity reactions, which include anaphylaxis, angioedema, and Stevens-Johnson syndrome, have been reported: ${ }^{7}$ however, it is difficult to quantify this because of the anecdotal nature of the self-reported cases. A recent case report proposed a link between rhabdomyolysis secondary to an interaction between sitagliptin and lovastatin. ${ }^{17}$ DPP-IV inhibitors are relatively new with respect to the use of other antidiabetic agents, and although sitagliptin use appears safe, additional side effects and drug interactions may become more evident with increased use of the medication.

Based on results from this study, an inappropriate initial dose of sitagliptin based on renal function was prescribed for $12.1 \%$ of patients ( $\mathrm{n}=35)$, with $21(60.0 \%)$ over-dosed and 14 patients (40.0\%) under-dosed. Patients who were seen by a nonpharmacist provider received a potentially inappropriate dose of sitagliptin $25.8 \%$ of the time for initiation of therapy. Only 1 patient with sitagliptin initiated by a pharmacist was given an inappropriate (under) dose.

\section{Limitations}

This study was a retrospective chart review that has many limitations. First, patients were not randomly assigned to the
TABLE 2 Rates of Inappropriate Initial Dosing a Sitagliptin by Pharmacist Versus Nonpharmacist

\begin{tabular}{l|c|c|c}
\hline & Pharmacist & Nonpharmacist & Total Sample \\
\hline & $\mathrm{n}=158$ & $\mathrm{n}=132$ & \\
\hline GFR $<30$ & $0 / 5$ & $4 / 7$ & $4 / 12$ \\
\hline $\begin{array}{l}\text { GFR between } \\
30 \text { and 50 }\end{array}$ & $0 / 11$ & $17 / 24$ & $17 / 35$ \\
\hline GFR $>50$ & $1 / 142$ & $13 / 101$ & $14 / 243$ \\
\hline Total & $1(0.6 \%)^{\mathrm{b}}$ & $34(25.8 \%)^{\mathrm{b}}$ & $35 / 290(12.1 \%)$ \\
\hline
\end{tabular}

aThe appropriate dose of sitagliptin per the label instructions is 100 milligrams $(\mathrm{mg})$ per day for patients with creatine clearance $(\mathrm{CrCl})>50$ milliliters $(\mathrm{ml})$ per minute, $50 \mathrm{mg}$ per day for patients with $\mathrm{CrCl} \geq 30-50 \mathrm{ml}$ per minute, and $25 \mathrm{mg}$ per day for patients with $\mathrm{CrCl}<30 \mathrm{ml}$ per minute. Glomerular filtration rate (GFR) was determined using the 4-point Modification of Dosing in Renal Disease equation. ${ }^{7}$ ${ }^{b} \mathrm{P}<0.001$ by Fisher's exact test.

pharmacist-managed diabetes management program versus usual care. Therefore, our study shows primarily that an opportunity exists in usual care to educate nonpharmacists about the prescribing information in the sitagliptin product label. However, it is also true that the adverse consequences in patients, or effects on intermediate biomarkers such as Alc, of not dosing sitagliptin according to renal function are unknown.

Second, GFR can fluctuate in individuals, so it is hard to know if a dose of sitagliptin was truly inappropriate at the time of initiation. The uncertainty associated with determination of precise renal function is accompanied by uncertainty in categorizing patients based on somewhat arbitrary cut-off points for dose changes. For example, 25 patients had a GFR that was within $1 \mathrm{ml}$ per minute of a range where a dosage adjustment would have been required by the label prescribing information for sitagliptin; these 25 patients were distributed nearly equally between the pharmacist group $(n=12)$ and the nonpharmacist group $(n=13)$.

Third, we used the MDRD formula, as now recommended by the National Kidney Foundation to estimate GFR for staging $\mathrm{CKD}^{14}$ and acknowledge that the sitagliptin prescribing information mentions that "creatinine clearance can be estimated from serum creatinine using the Cockroft-Gault formula."' We relied on MDRD because every basic metabolic profile at our medical clinic includes a GFR value calculated by the 4-point MDRD equation as part of an initiative to proactively identify patients with CKD. Use of the MDRD equation has not been validated as a reliable estimation of renal function in monitoring drugs. ${ }^{15} \mathrm{Few}$ studies exist evaluating the comparison of the Cockroft-Gault equation with the MDRD equation in drug dosing..$^{18-20}$ Therefore, use of the MDRD equation represents current practice trends in our medical clinic but may not represent common practice in other medical facilities when adjusting medications according to patient renal function.

Fourth, the present study did not evaluate outcomes associated with presumably inappropriate dosing of sitagliptin. The 


\section{Drug Use Evaluation of Sitagliptin Dosing by Pharmacist Versus Nonpharmacist Clinicians in an Internal Medicine Department of a Private Physician-Owned Multispecialty Clinic}

lack of outcomes data for Alc levels or actual patient harm associated with over-dosing or under-dosing according to renal function means that it is not possible from this study to determine if the dosing of sitagliptin was truly clinically inappropriate.

\section{Conclusion}

To our knowledge, this study is the first to investigate the initial dosing of sitagliptin prescribed by a pharmacist compared with nonpharmacist prescribers based on the prescribing information in the product label. The results from this study reveal that there is an apparent opportunity to improve the adherence of initial sitagliptin dosing to the label recommendations for dose adjustment according to renal function. Compared with nonpharmacist prescribers, patients who had sitagliptin dosing initiated by a pharmacist were more likely to receive the appropriate dose based on renal function.

\section{Authors}

M. SHAWN MCFARLAND, PharmD, was Clinical Ambulatory Care Pharmacist and Assistant Professor, Murfreesboro Medical Clinical and University of Tennessee College of Pharmacy, Murfreesboro, Tennessee, when this research was conducted. He is now Clinical Pharmacist Specialist in Primary Care and Assistant Professor, Alvin C. York Veteran Hospital and University of Tennessee College of Pharmacy, Murfeesboro, Tennessee; L. BRIAN CROSS, PharmD, is Clinical Ambulatory Care Pharmacist and Associate Professor, Holston Medical Group and University of Tennessee College of Pharmacy, Kingsport, Tennessee; BENJAMIN GROSS, PharmD, is Clinical Ambulatory Care Pharmacist and Assistant Professor, Holston Medical Group and University of Tennessee College of Pharmacy, Kingsport, Tennessee; CHAD GENTRY, PharmD, is PGY-1 Resident, Holston Medical Group and University of Tennessee College of Pharmacy, Kingsport, Tennessee; JEFFREY TUNNEY, PharmD, is PGY-1 Resident, Tennessee Valley Health Care System, Nashville, Tennessee; and U.P. PATEL, MD, is Internal Medicine Physician, Murfreesboro Medical Clinic, Murfreesboro, Tennessee.

AUTHOR CORRESPONDENCE: M. Shawn McFarland, PharmD, Alvin C. York Veterans Administration Hospital, 3400 Lebanon Pike, Murfreesboro, TN 37130. Tel.: 615.867.6000, ext. 24796; Fax: 615.225.4801; E-mail: Michael.McFarland2@va.gov.

\section{DISCLOSURES}

There was no external funding for this research. This research was presented in part as a poster at the American College of Clinical Pharmacy meeting on April 24, 2009, in Orlando, Florida.

The concept and design were contributed primarily by McFarland. Cross collected the data with the assistance of Gross and Tunney. McFarland interpreted the data and wrote the manuscript with the assistance of Gentry and Tunney. Gentry, McFarland, Patel, and Tunney revised the manuscript.

\section{ACKNOWLEDGEMENT}

The authors would like to thank Ping Zhang, PhD, for her contribution in statistical analysis for this manuscript.

\section{REFERENCES}

1. Centers for Disease Control and Prevention. National Diabetes Fact Sheet: general information and national estimates on diabetes in the United States, 2007. Atlanta, GA: U.S. Department of Health and Human Services, Centers for Disease Control and Prevention, 2008. http://www.cdc.gov/diabetes/ pubs/pdf/ndfs_2007.pdf. Accessed August 13, 2008.

2. Nathan, D. Finding new treatments for diabetes-how many, how fast how good? N Engl J Med. 2007;356(5):437-40.

3. Raz I, Hanefeld M, Xu L, et al. Efficacy and safety of the dipeptidyl peptidase-4 inhibitor sitagliptin as monotherapy in patients with type 2 diabetes mellitus. Diabetologia. 2006;49(11):2564-71.

4. Aschner P, Kipnes MS, Lunceford JK, et al. Effect of the dipeptidyl peptidase-4 inhibitor sitagliptin as monotherapy on glycemic control in patients with type 2 diabetes. Diabetes Care. 2006;29(12):2632-37. Available at: http://care.diabetesjournals.org/content/29/12/2632.full.pdf+html. Accessed August 10, 2009.

5. Charbonnel B, Karasik A, Liu J, et al. Efficacy and safety of the dipeptidyl peptidase-4 inhibitor sitagliptin added to ongoing metformin therapy in patients with type 2 diabetes inadequately controlled with metformin alone. Diabetes Care. 2006;29(12):2638-43.

6. Hermansen K, Kipnes M, Luo E et al. Efficacy and safety of the dipeptidyl peptidae- 4 inhibitor, sitagliptin, in patients with type 2 diabetes mellitus inadequately controlled on glimepiride alone or on glimepiride and metformin. Diabetes Obes Metab. 2007;9(5):733-45.

7. Merck \& Co. Januvia (sitagliptin) package insert. Whitehouse Station, NJ; 2007. Available at: http://www.merck.com/product/usa/pi_circulars/j/januvia/januvia_pi.pdf. Accessed August 10, 2009.

8. Bergman AJ, Stevens C, Zhou Y, et al. Pharmacokinetic and pharmacodynamic properties of multiple oral doses of sitagliptin, a dipeptidyl peptidaseIV inhibitor: a double-blind, randomized, placebo-controlled study in healthy male volunteers. Clin Ther. 2006;28(1):55-72

9. Herman GA, Stevens C, Van Dyck K, et al. Pharmacokinetics and pharmacodynamics of sitagliptin, an inhibitor of dipeptidyl peptidase IV, in healthy subjects: results from two randomized, double-blind, placebo-controlled studies with single oral doses. Clin Pharmacol Ther. 2005;78(6):67588 .

10. Herman GA, Bergman A, Liu F, et al. Pharmacokinetics and pharmacodynamic effects of the oral DPP-4 inhibitor sitagliptin in middle-aged obese subjects. J Clin Pharmacol. 2006;46(8):876-86.

11. Vincent SH, Reed JR, Bergman, AJ, et al. Metabolism and excretion of the dipeptidyl peptidase 4 inhibitor [14C] sitagliptin in humans. Drug Metab Dispos. 2007;35(4):533-38. Available at: http://dmd.aspetjournals.org/cgi/ reprint/35/4/533. Accessed August 10, 2009.

12. Bergman AJ, Cote J, Yi B, et al. Effect of renal insufficiency on the pharmacokinetics of sitagliptin, a dipeptidyl peptidase-4 inhibitor. Diabetes Care. 2007;30(7):1862-64.

13. Chan JC, Scott R, Arjona Ferreira JC, et al. Safety and efficacy of sitagliptin in patients with type 2 diabetes and chronic renal insufficiency. Diabetes Obes Metab. 2008;10(7):545-55.

14. National Kidney Foundation. K/DOQI clinical practice guidelines for chronic kidney disease: evaluation, classification and stratification. Am J Kidney Dis. 2002;39(2 Suppl 1):S1-266. 
Drug Use Evaluation of Sitagliptin Dosing by Pharmacist Versus Nonpharmacist Clinicians in an Internal Medicine Department of a Private Physician-Owned Multispecialty Clinic

15. Rigalleau V, Lasseur C, Perlemoine C, et al. Estimation of glomerular filtration rate in diabetic subjects: Cockcroft formula or modification of Diet in Renal Disease study? Diabetes Care. 2005;28(4):838-43. Available at: http:// care.diabetesjournals.org/content/28/4/838.full.pdf+html. Accessed August 10, 2009.

16. Nathan DM, Buse JB, Davidson MB, et al. Medical management of hyperglycemia in type 2 diabetes: a consensus algorithm for the initiation and adjustment of therapy: a concensus statement of the American Diabetes Association and the European Association for the Study of Diabetes. Diabetes Care. 2009;32(1):193-203. Available at: http://care.diabetesjournals.org/content/32/1/193.full.pdf+html. Accessed August 10, 2009.
17. DiGregorio R, Pasikhova Y. Rhabdomyolysis caused by a potential sitagliptin-lovastatin interaction. Pharmacotherapy. 2009;29(3):352-56.

18. Golik M, Lawrence K. Comparison of dosing recommendations for antimicrobial drugs based on two methods for assessing kidney function Cockcroft-Gault and Modification of Diet in Renal Disease. Pharmacotherapy. 2008;28(9):1125-32.

19. Shord S, Bressler L, Radhakrishnan L, Ningyu Chen, Villano J. Evaluation of the Modified Diet in Renal Disease equation for calculation of carboplatin dose. Ann Pharmacother. 2009;43(2):235-41.

20. Hermsen ED, Maiefski M, Florescu MC, Qiu F, Rupp ME. Comparison of the Modification of Diet in Renal Disease and Cockcroft-Gault equations for dosing antimicrobials. Pharmacotherapy. 2009;29(6):649-55. 